Elemente und Ephemeride des Cometen 1890 ... (Spitaler Nov. 16).

Aus meinen Beobachtungen des Cometen am 16. November, 8. December und 29. December 1890 erhielt ich mit Rulcksicht auf Parallaxe und Aberration folgende Elemente, die den mittleren Ort im Sinne Beob. - Rechn. auf $\mathrm{d} \alpha \cos \beta=+\mathrm{I}: 8, \mathrm{~d} \beta=+0.6$ derstellen.

$$
\begin{aligned}
& T=1890 \text { Oct. } 26.60123 \text { M.Z. Berlin } \\
& x=5^{\circ} 25^{\prime} 5^{8.2} \text { ) } \\
& \delta=455 \text { 5 I.7 M. Aequ. } 1890.0 \\
& i=125044.5 \\
& \Phi=28^{\circ} 7^{\prime} 39^{\circ} 4 \\
& \log a=0.536478 \\
& \mu=556: 240 \\
& U=6.3785 \text { Jahre }
\end{aligned}
$$

Heliocentrische Aequatorealcoordinaten fur 1891.0 .

$$
\begin{aligned}
& x=[9.994545] r \cdot \sin \left(v+147^{\circ} 43^{\prime} 14^{\prime \prime 6}\right) \\
& y=[9.927758] r \cdot \sin (v+632819.9) \\
& z=[9.744159] r . \sin (v+435258.5)
\end{aligned}
$$

Hieraach hat Herr Rosmanith die folgende Epbemeride fur 1 a $^{\mathrm{h}}$ M. Z. Berlin berechnet:

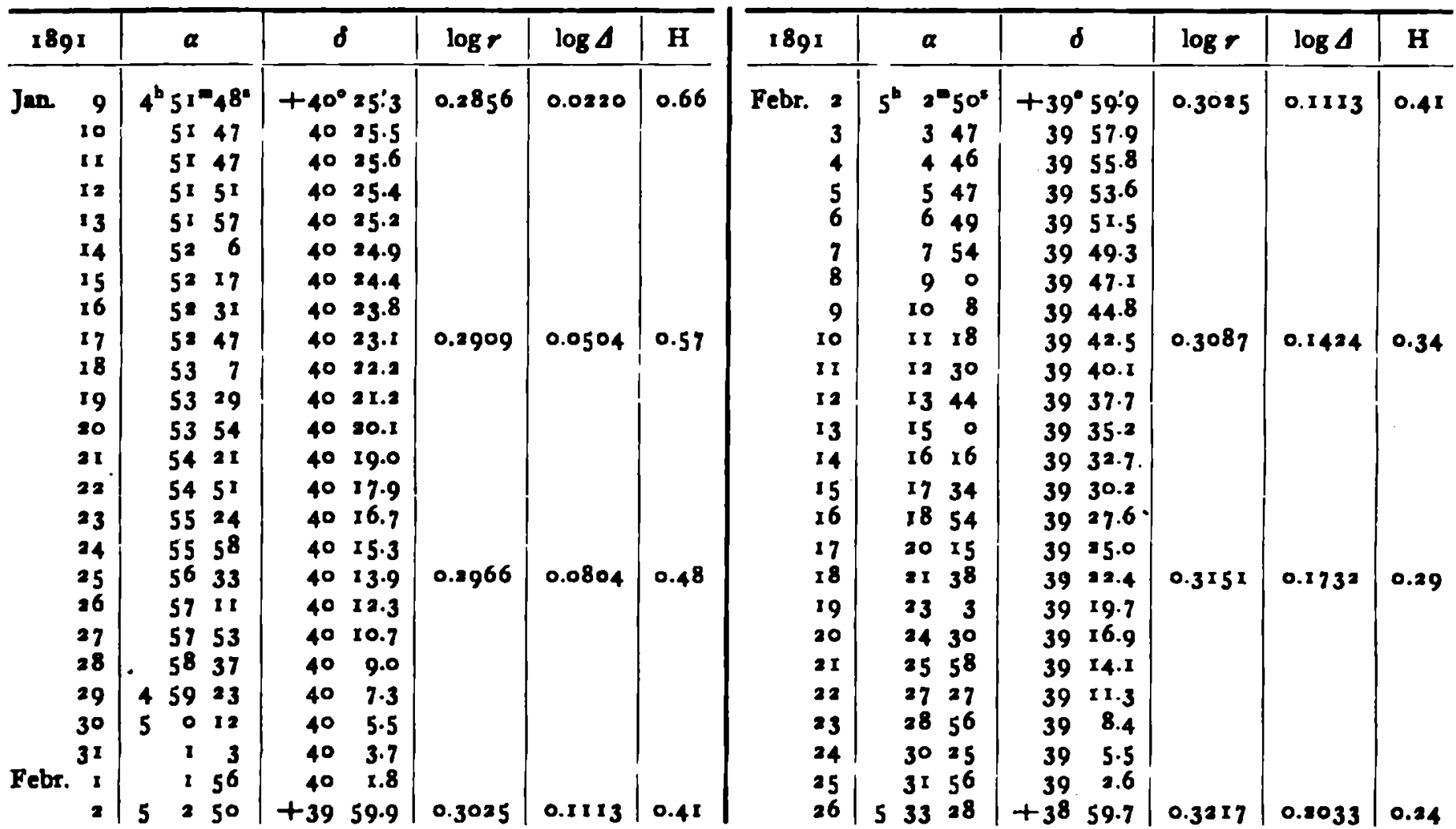

Als Einheit der Helligkeit ist die sur Zeit der Entdeckung gewihlt.

Sternwarte Wied-Wuhring 1891 Jan. 3 .

R. Spitaler.

\title{
Ephemeride des Winnecke'schen Cometen für die Opposition 1891.
}

Durch Nr. 225 des Astr. Journ., in welches ich leider erst vor 4 Tagen, anlusslich meines Ferienaufenthalts in Wien, Einblick nehmen konnte, wurde ich auf das Anerbieten Herrn Barnard's, den Winnecke'schen Cometen auch in seiner grossen Entfernung aufsuchen zu wollen, aufmerksam. Da die Störungsrechnung bis zum nilchsten Periheldurchgang (1892 Ende Juni) bereits fertig gestellt ist, konote ich seinem daselbst ausgesprochenen Wunsch nach einer genaues Ephemeride leicht entsprechen.

Die unten folgende Ephemeride, aus der man die verhulltnissmussig gunstigen Sichtbarkeitsverhaltmisse dieset Cometen ersieht, durfte dea Ort desselben innerhalb einiger Bogensecunden genau angeben. 
Elemente des Cometen.

Epoche und Osc. 189 I Januar I 1 .0 M. Z. Berlin

$$
\begin{aligned}
& M=269^{\circ} \quad 4^{\prime} 32^{\prime \prime} \text { I }^{\prime} \\
& \pi=276 \text { II } 32.07 \\
& \omega=104 \quad 5 \quad 46.19 \\
& i=143 \mathrm{r} 5 \mathrm{I} .37 \\
& \varphi=46335 \mathrm{I.13} \\
& \mu=609^{\prime \prime 7487572}
\end{aligned}
$$

\begin{tabular}{|c|c|c|c|c|c|c|c|c|}
\hline 1891 & \multicolumn{3}{|c|}{$\alpha$} & \multicolumn{3}{|c|}{$\delta$} & $\log r$ & $\log 4$ \\
\hline Jan. & & $22^{\mathrm{D}}$ & m $\quad$ r & $+24^{\circ}$ & $23^{\prime}$ & $16 "$ & 0.66246 & 0.56 I26 \\
\hline 8 & & & I 3.7 & 24 & 27 & 20 & 0.66208 & 0.56030 \\
\hline 9 & & 20 & 25.0 & 24 & $3 I$ & 24 & 0.66170 & 0.55938 \\
\hline 10 & & I 9 & $35: 8$ & 24 & 35 & 28 & 0.66 I $3^{2}$ & $0.55^{8} 5^{\circ}$ \\
\hline I I & & 18 & $46 . I$ & 24 & 39 & 3 I & 0.66094 & 0.55766 \\
\hline 12 & & 17 & 55.9 & 24 & 43 & 34 & 0.66056 & 0.55686 \\
\hline I 3 & & I 7 & 53 & 24 & 47 & 37 & 0.66018 & 0.55610 \\
\hline 14 & & 16 & 14.3 & 24 & 51 & 39 & 0.65979 & $0.5553^{8}$ \\
\hline I 5 & & I 5 & 22.8 & 24 & 55 & 39 & $0.6594 I$ & 0.55469 \\
\hline I 6 & & I 4 & 30.9 & 24 & 59 & 37 & 0.65902 & 0.55404 \\
\hline 17 & & I 3 & 38.7 & 25 & 3 & 33 & 0.65863 & 0.55343 \\
\hline I 8 & 8 & I 2 & 46.3 & +25 & 7 & 27 & 0.65825 & 0.55286 \\
\hline
\end{tabular}

\begin{tabular}{|c|c|c|c|c|c|c|c|c|c|}
\hline \multicolumn{2}{|c|}{ I 891} & \multicolumn{3}{|c|}{$\alpha$} & \multicolumn{3}{|c|}{$\delta$} & $\log r$ & $\log \Delta$ \\
\hline Jan. & 19 & & & $53^{s .8}$ & $+25^{\circ}$ & ${ }^{\circ}$ I I I' & $19^{\prime \prime}$ & 0.65786 & 0.55233 \\
\hline & 20 & & & 1.2 & 25 & I 5 & 8 & $0.6574^{6}$ & 0.55184 \\
\hline & $2 \mathrm{I}$ & & o & 8.4 & 25 & 18 & 55 & 0.65707 & 0.55139 \\
\hline & 22 & & & 15.4 & 25 & 22 & 40 & 0.65668 & 0.55098 \\
\hline & 23 & & & $22 \cdot 3$ & 25 & 26 & 23 & 0.65628 & $0.5506 \mathrm{I}$ \\
\hline & 24 & & & 29.2 & 25 & 30 & 4 & 0.65588 & 0.55028 \\
\hline & 25 & & & 36.1 & 25 & 33 & 43 & 0.65548 & 0.54999 \\
\hline & 26 & & & 43.0 & 25 & 37 & 20 & 0.65508 & 0.54974 \\
\hline & 27 & & 4 & 49.9 & 25 & 40 & $5^{2}$ & 0.65468 & 0.54954 \\
\hline & 28 & & 3 & 56.8 & 25 & 44 & 20 & 0.65427 & 0.54939 \\
\hline & 29 & & 3 & 4.0 & 25 & 47 & 45 & $0.653^{87}$ & 0.54929 \\
\hline & 30 & 8 & 2 & I 1.6 & +25 & $5 I$ & 7 & 0.65347 & $0.549^{2} 3$ \\
\hline
\end{tabular}

Ephemeride für $12^{\text {h }}$ M. Z. Berlin.

Opposition in AR.: 1891 Jan. 20.8 M. Z. Berlin.

Eine Abschrift der Ephemeride ist bereits gestern den Herren Barnard, Perrotin und Palisa ubersandt worden.

Wien I891 Jan. 2.

Dr. Eduard Frhr. v. Haerdtl.

\section{Elemente und Ephemeride des Planeten (253) Mathilde.}

Der Planet wurde 1885 Nov. 12 von Herrn Dr. J. Palisa in Wien entdeckt und in der ersten Erscheinung bis

\begin{tabular}{|c|c|c|c|c|c|c|}
\hline & & $\Delta \alpha$ & $\Delta \delta$ & & $\Delta \alpha$ & $\Delta \delta$ \\
\hline 1885 & Nov. 12 & -3.2 & +4.5 & I 886 Jan. $3 \mathrm{I}$ & $+1: 8$ & -o."6 \\
\hline & Dec. 4 & 0.0 & -2.9 & r 887 Jan. 29 & +0.4 & $-x .7$ \\
\hline I 886 & Jan. & +0.3 & -3.8 & & & \\
\hline
\end{tabular}
I 886 Febr. 8 achtmal beobachtet, nämlich sechsmal in Wien und zweimal in Berlin; die Pariser Meridianbeobachtung vom 8. December 1885 betrifft indessen den Planeten nicht. In der zweiten Erscheinung wurde der Planet nur einmal und zwar in Wien beobachtet. - Die Elemente wurden erhalten durch Verbesserung der Elemente im Berliner Jahrbuch für 1892 nach der Tietjen'schen Methode. Eine Nachrechnung der fünf theilweise nur auf je einer Beobachtung beruhenden Normalörter ergab die Abweichungen (Beob._- Rechn.):

Die Störungen durch Jupiter während der Jahre 1887 bis $189 \mathrm{I}$ wurden in Intervallen von 80 Tagen berechnet.

Elemente :

Epoche und Osculation: 189 an. 31.0 M. Z. Berlin.

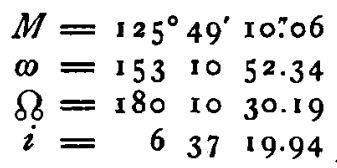

$\varphi=15^{\circ} 14^{\prime} 21^{\prime \prime} 79$

M. Aequ. 1891.0

$\mu=822.82460$

$\log a=0.4231329$

Heliocentrische Aequatorealcoordinaten für I891.0.

$$
\begin{aligned}
& x=[0.00000] r \cdot \sin \left(v+63^{\circ} 21: 30\right) \\
& y=[9.98098] r \cdot \sin (v+33320.93) \\
& z=[9.46173] r \cdot \sin (v+33325 \cdot 30)
\end{aligned}
$$

